\title{
Re-engineering Workflows: Changing the Life Cycle of an Electronic Health Record System
}

\author{
Jane M. Brokel, Ph.D., RN ${ }^{1^{*}}$, Sheila Ochylski, RN, MS ${ }^{2}$, \\ J. Michael Kramer, MD, MBA ${ }^{3}$ \\ ${ }^{1}$ Assistant Professor, College of Nursing, University of Iowa, Iowa City, IA, USA \\ ${ }^{2}$ Director of Clinical Transformation, Clinical Operations Improvement, Trinity \\ Health - Novi, MI, USA \\ ${ }^{3}$ Chief Medical Information Officer, Trinity Health - Novi, MI, USA
}

Submitted June 2010. Accepted for publication May 2011.

\begin{abstract}
An existing electronic health record (EHR) system was re-engineered with cross-functional workflows to enhance the efficiency and clinical utility of health information technology. The new designs were guided by a systematic review of clinicians' requests, which were garnered by direct interviews. To design cross-functional, patient-centered workflows, several multidisciplinary teams from the health system of hospitals, clinics, and other services participated. We identified gaps and inconsistencies with current care processes and implemented changes that improved workflow for patients and clinicians. Our findings emphasize that, to coordinate care between many providers, process workflow must be standardized within and across settings and focus on patient care processes, not the technology. These new, comprehensive, admission-todischarge workflows replaced the older, functional- and departmental-process flow charts that had fallen short. Our experience led to integrated redesign of the workflows, review prior to implementation and ongoing maintenance of this process knowledge across 37 hospital facilities.
\end{abstract}

Keywords: health informatics, health information technology, patient-centered, reengineering workflows, issue resolution

\section{INTRODUCTION}

The use of electronic health records (EHRs) has been touted as a powerful new tool to improve patient care and reduce morbidity and mortality. In reality, however, implementation of this new technology requires attention to workflow details to improve utility of EHRs [1-4]. Yet, it should not be surprising that mishaps in patientcare will occur when a diverse collection of disciplines, patient/family units, and

*Corresponding author: Jane M. Brokel, 483 NB - 50 Newton Road, The University of Iowa, Iowa City, IA 52242, USA. Phone: (319) 335-7111, Email: jane-brokel@uiowa.edu; jbrokel@gmail.com. Other authors: Ochylsks@trinity-health.org; jmichaelkramer@gmail.com. 
caregivers interact with health information technology (HIT); indeed, with the promise of supporting each comes the potential to introduce errors [5]. Clinicians will find ways to care for patient with and without full use of the EHR capabilities [6]. The objective of this paper is to report the results of re-engineering an existing EHR system with cross-functional, patient-centered workflows to enhance the efficiency and clinical utility of health information technology. Our purpose was to improve the utility of EHRs by tailoring workflows and use of technical design to meet the needs of the users. Workflows are defined here as the sequence of ordered steps in a clinical process that constitutes patient care. During our first 6 implementations, we used rudimentary and modular workflows. Between 2005 and 2011, we introduced and then standardized integrated workflows for the subsequent 31 additional health system's EHR implementations. More clinicians were involved to establish the new workflows locally and system-wide and remove any gaps prior to EHR implementations.

\subsection{Scientific Background}

The Institute of Medicine report, "To Err is Human" [7] was a call to action. Although the use of EHRs is advocated to improve patient safety, recent reports show that nonprescribers using computerized physician order-entry (CPOE) systems are prone to making unintended errors $[8,9]$. Indeed, although physician groups, and other key stakeholders, see merit in electronic records, they also recommend testing applications with different patient populations so the systems can be implemented safely [10].

Theoretically, converting from paper-based to electronic health information systems has far-reaching benefits: it should reduce paper work, duplicate testing [11], and medication errors [12]; it should prevent conflicting courses of treatment [13], shorten waiting-room time [14] and speed lab test results [15]. Despite these advantages, experts have questioned whether EHR implementations have, in reality, improved patient outcomes and safety. A number of studies show mixed results, while others show that morbidity and mortality declined $[16,17]$. Therefore, we need to understand fully how the daily processes and associated workflows, for different types of patient populations, are inter-related. The impact of any changes will differ because health organizations are at different technical and organizational starting points in terms of computerizations, knowledge resources, and organizational culture $[18,19]$.

When technology lacks "data capture" for information [18] (e.g., it may be missing an assessment section for Gordon's Functional Health Patterns [20], or new treatment protocols, or a patient-education topic) or is limited in function (e.g., it cannot link indications to medications or lab results within view when ordering), it fails the nurse or physician in making decisions. To bridge the gap left by the EHR, the clinician's workflow will need to devise a way (workaround) $[5,18]$ to provide evidence-based, safe care despite the flaw in the EHR [18]. The workarounds have been described as gaps in social technical interactions with health information technology, which have been linked to unintended consequences [5] with missed or unclear communication or missed or delayed care. To understand precisely where the flaws in the EHR originate, we looked to the processes for delivery of services. 
For decades, modeling flow charts have been used to represent quality improvement activities (for our purposes, workflows). To improve quality and efficiency, vendors and consultants also have used workflows in a modular approach (i.e., task specific steps like entering orders) to handle EHR adoption. Although modular workflows have improved for clinicians the handling of information for specific chronic diseases [21], the patient's holistic perspective and preferences have largely been absent in this design [22]. Instead, the modular workflow focused more on applications for ordering medications or documenting care and focused less on care delivery, which includes decision making to diagnose, plan, evaluate and coordinate with the interdisciplinary team. To improve how EHRs manage information for decision making and coordination of care, patient-centered workflows have been devised.

Our aim was to change the workflow processes so they reflected a holistic approach to patient care. Over the past five years, we have examined how to use EHRs to complement patient-centered workflows. The challenge was to maximize both efficiency and quality within the patient-centered workflows with a focus on the patient-provider interactions and evidence-based decisions. There was and continues to be a critical need for standardization of workflow to transfer knowledge from one hospital and clinic implementation to the next implementation. This would save time and effort with limited human resources.

In this study, the desire for patient-centered workflows evolved from a health system's need to improve care (with or without the support of EHR system). The study occurred with the $4^{\text {th }}$ largest Catholic health system in the United States. This system employs 45,000 full-time equivalents, with more than 8,000 active physicians associated with 19 ministry organizations, encompassing 45 hospitals, 379 ambulatory clinics and facilities, 29 long-term care facilities, home-health and hospice programs, in seven states. The health system HIT services are coordinated under one infrastructure and directly aligned under the leadership of a chief information officer and a chief medical informatics officer. The system's clinical leadership was decentralized among the chief nurse officers and chief medical officers, yet unified through the support from a corporate, clinical-operations improvement department of 20 persons, along with finance and information systems staff. The health system began implementing a comprehensive commercial EHR in 2001. Seventeen of the nineteen ministry organizations use common HIT applications, which include computerized physician order entry, electronic health records, nursing documentation (with wireless devices), emergency-department event tracking, physician documentation, adverse drug event alerts, and clinical decisionsupport logic modules. In many of the organizations, additional applications include radiology and lab systems, anesthesia module, surgery module and ambulatory office modules, and phone triage tracking. In 2002, modular workflows were introduced to prepare staff for implementations. In 2005, patient-centered workflows were introduced shortly before the $6^{\text {th }}$ EHR implementation site. The $6^{\text {th }}$ implementation site was studied because both modular and patient-centered workflows were used. A majority of the hospital clinicians reviewed processes using the modular workflows while the Emergency Department was introduced to the first patient-centered 


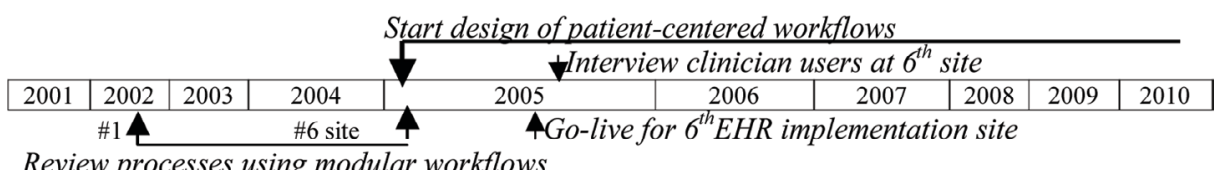

Review processes using modular workflows

Figure 1. Transition of reviewing processes from modular to patient-centered workflows

workflow to review their processes. Figure 1 shows the timeline for the transitions from reviewing processes with modular workflow to review processes with comprehensive patient-centered workflows.

\section{METHODS}

We explored clinician requests for change, after they had used an EHR in their natural settings for six weeks. The EHR system included applications for clinical documentation, CPOE, physician inbox, profiling for dictation review and coding, electronic medication administration record, laboratory and diagnostic results viewing, clinical decision support alerts and reminders, pharmacy, radiology and emergency room event tracking systems. Sixteen physicians and over one hundred end-users, from eight clinical departments and eleven nursing units, participated in group interviews. The post-implementation interviews were conducted by a team of nine, including an informatics physician, pharmacist, nurse and HIT staff. During the first two days, the team invited groups of clinicians and support staff for 30 to 60 minute sessions, to discuss gaps within the modular workflows and how to improve the workflow. (The emergency services group was the exception, since it implemented and used patientcentered workflow for the first time.)

Next, the team split up and visited clinicians on patient floors and in departments, asking a list of semi-structured questions. All disciplines were approached, in all units and departments; most accepted the invitation to participate. The anonymous, random comments from interviews were collected within Excel spreadsheets, and issues and requests recorded into the online tracking system. The HIT staff reviewed and sorted the comments as content changes, role changes, process changes, or requests to change the technology. The online tracking system provides a way to routinely evaluate system and organization changes, in routine monthly reports. These reports were used to describe the pre- and post-implementation changes. The Institutional Review Board at the implementation site had approved the research activities.

Because the patient-centered workflows reduced the incidence of gaps with implementations, we describe the design of our standard workflows in Table 3, which model data flow and information used in patient's interactions during registration, admission, daily assessments, care coordination, planning and discharge, account management, and regulatory reporting. We provide an example of components for one of the fourteen types of patient-centered workflows in Figure 2. We will also give an example of what was standardized for patients having surgery and how clinicians' use of technology across system facilities was central to patients' care. 


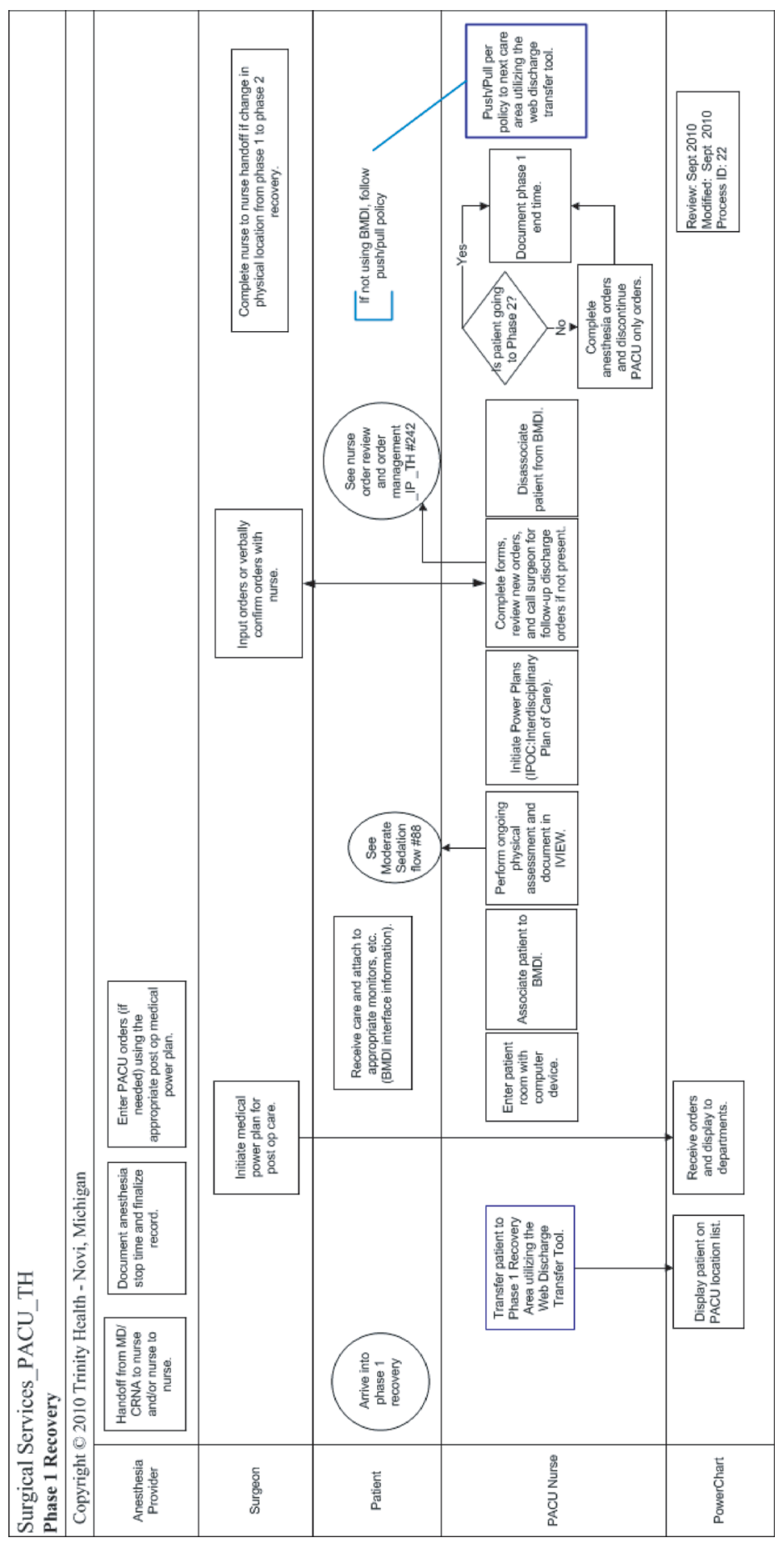

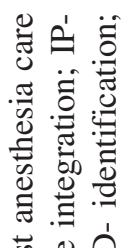

苍.

b文芒

苂

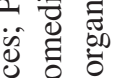

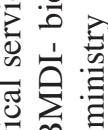

on

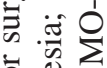

它过

苍总

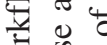

苛壳

गृ

可 $\cong$

कृ

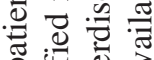

․ㅡㄹ

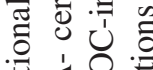

离宏䓃

导西兰 응

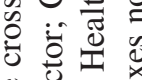

용용

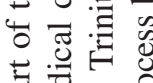

氞焉志

范它完

를 苛

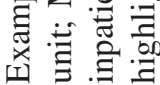

ن 
Table 1. Major processes, sub-processes, HIT systems, and modular workflows used in change management

\begin{tabular}{lcccc}
\hline \multicolumn{1}{c}{ Major processes } & $\begin{array}{c}\text { Patient } \\
\text { access }\end{array}$ & $\begin{array}{c}\text { Care } \\
\text { delivery }\end{array}$ & $\begin{array}{c}\text { Service } \\
\text { document }\end{array}$ & $\begin{array}{c}\text { Revenue } \\
\text { collection }\end{array}$ \\
\hline Number of sub processes & 8 & 9 & 9 & 12 \\
Number of HIT systems & 4 & 3 & 2 & 4 \\
Number of workflow pages & 29 & 69 (496 all services) & 28 & 32 \\
\hline
\end{tabular}

\section{RESULTS}

At the organizational level overall, clinicians were hindered with the disjointed approach of the modular workflows, which focused on the technology, rather than on care process and providing information to the end users: patients, clinicians, and administrators. Table 1 displays the framework for the functions these modular workflows addressed, in 2002-2004, showing that several important decision processes were absent. In 2005, however, one group from Emergency Services was satisfied; significantly, because its workflow was a patient-centered workflow. This department had minimal concerns during implementation, a fact that was noticed in the tracking of issues. In other departments, however, clinicians and support staff called for greater standardization and integration of the functions, and a closer representation of daily activities in patient care.

\subsection{Suggestions Resulted in Changes in Workflow Modeling}

System users identified four points that they thought were crucial to consider and these suggestions resulted in changes in the approach to workflow modeling and review process in future implementations:

- Users need tutorials and training prior to process review-One participant commented, "There was a lot of missing information during process review (i.e., learning modular workflows for EHR applications) that, had we seen the system to begin with, we could have avoided a lot of pitfalls." Therefore, tutorials and resolution plans could be created that close gaps in interactions with technology. When functions of the EHR are being viewed, real-time tutorials could provide a standard way to highlight potential gaps in the workflow. In response, we created patient-centered workflows as resolution plans in 2005 for each service line and identify clinical service line leaders who participate in early advanced training on the system. Now, any classroom tutorial will address the patient-centered processes.

- Allow time to review the process-A participant stated, "Take advantage of process review; it's the time to assess the extraneous parts of your processes so you can remove them and not deal with them in the electronic world." Teams need to know that process reviews can be tedious, but that they are necessary to get the most out of the new technology. In response, we facilitated high-level preimplementation process review during the 14 months prior to implementation, and subsequently detailed drill down work sessions. These activities and feedback on gaps are tracked by the project management team and now occur reliably for every organization. 
- Identify process liaison, service line leaders, and super-users early-From our interviews, we learned that the process review had been very difficult for participants. Because clinician leaders and super-users were not trained in EHR functionality first. In response, we created expectations that any patient-centered workflow is an ongoing methodology in the organization and should leverage the organizational process roles and approaches such as LEAN or Six Sigma. A dedicated role of "process liaison" assures that the organization maintains attention to process before and after implementation. Exposure to the EHR and subsequent training should occur before process review of future redesigned workflow to establish both cognitive and functional understanding. These process experts should be chosen at least one year before implementation. Service line leader are well regarded operational leader who represent the major service lines and are key stakeholders involved in process review. Their process and system knowledge will be essential to deploying the process change and subsequent adoption in each area of the organization. Finally, clinical informatics experts should be present when process workflows are reviewed by clinicians and support staff who are super-users or leaders.

- Teach end users how the workflow changes and why-One participant commented, "we need a better understanding of the 'Why's'." Indeed, a team cannot build on the strengths of the EHR if they don't know what they are.

\subsection{Requests and Comments from Professionals}

Each professional group had a unique and valuable point of view that provided insight on how to enhance workflow. Below are typical examples of the advice we followed.

Physicians: This group encouraged us to "Take a grassroots approach with your computerized physician order entry (CPOE) team and include physician leads from obstetrics, emergency department, surgery, psychiatry, family practice, pediatrics, and hospitalists." Before implementation, physicians were able to disseminate the information to their specialties for six months. Thus, early on, the clinical-informatics staff had meaningful discussions with a group of key physicians. Users liked this approach, and gave responses such as, "The extent to which physicians were brought into the process was done well." Some suggested that the organization should establish order sets, in a collaborative way, across specialties and disciplines, so that all parties are involved.

Another request was to "portray in training that the EHR is an opportunity for improvement rather than a forced change." We envision that a month before implementation, clinician training should include more role-playing (with workflow). In addition, physicians should be trained in terms of their own specialties, thus "make it personal so the physicians can understand what's in it for them." A workflow for downtime was also requested to allow users to know what to do when there is downtime.

Pharmacists: To allow for a period of adjustment, one pharmacist suggested that, before implementation, the department should test the new system on fifty medication orders each week for two weeks. This would allow them to practice with a large number of drug products and order types to identify successes and problems.

Nurses: Nurses asked that trainers be clear and specific about the classification and organization of fields, tabs, folders; they wanted to be taught exactly what the end users will use in daily workflows. For example, when using the medication profile function, there was no way to document and find when the patient last took the medication at 
home. In addition, when patient education was documented, the information might be erroneously displayed later, at discharge. While the nurses appreciated the ability to document assessments and automate notifications to other team members from clinical decision support, many wanted to be able to document nursing diagnoses, patient outcomes and plan the patient's care electronically. They also wanted a way to link directly with nursing interventions to document.

Ancillaries: Therapists, dieticians, and others stated the trainers never provided a workflow to guide documentation. Trainers need to answer specific questions about the EHR such as how orders provide a planned frequency within a therapist's workflow.

Leaders: The nurse informatics specialists and manager suggested, "Determine in advance what to document, where to document, and include the workflow in the training." Additionally, terminology should not be unfamiliar; otherwise finding procedures, interventions and diagnostic tests is challenging. Clearly, functions for documentation should be similar in all EHR applications, and they should allow information to be reused; furthermore, the documentation should follow the nursing processes, medical processes or rehab processes which are all focused on a patientfamily unit and decisions about care.

\subsection{Health System Standardization}

At the system level, their EHR system was implemented in seven states, from 20012010 , and in two phases. The evolution led to many points for change in practice. Not all applications were fully deployed in all settings, but approximately eighty-five percent are utilizing EHR applications described earlier in methods section. About 67,000 users (including records and finance staff) were generating over 54 gigabytes per month, contributing to over 8.5 million patient longitudinal records, over the past eight years. Post-implementation interviews, conducted in 2003 and 2004, prompted the redesign and integration of workflow processes. A one-page executive workflow was used to outline the patient care processes from patient's initial chief complaint for admission, through assessments, diagnostic testing, diagnosis and a plan for care for medication management, interventions and treatment, to evaluation of progress and finally transition of care. By 2010, the health system had inventoried and stored, within a process management database, 224 workflows categorized into twenty-five servicerelated groups (see Table 2 for service lines with patient-centered workflows). The database can track the owners and versions. A typical patient-centered workflow is the example of a patient receiving surgical services. The workflow for ambulatory, emergent, and inpatients includes nineteen components (see Table 3 for patient-centered flows and Table 4 for department-specific flows).

Before the surgical procedure, the workflows included the patient's participation in lab testing and follow-through to give necessary information to the nurses, anesthesia provider and surgeon. During this time, the patient needed to be a major contributor to the information flow. During the intra-operative workflows, the patient is supported by an anesthesia provider, operating room nurse, surgeon and technician. The workflow details included CPOE processes with order sets for diagnostic tests (specimens, radiology), supplies, medications, irrigations, and blood administrations. The recovery 
Table 2. Service lines with patient centered workflows and department-specific needs for workflow.

\section{Patient-centered workflows with integrated processes}

Emergency services walkin and ambulance arrival

Hemodialysis services

Medical inpatient services

Ambulatory clinic services

\section{Number of workflows used with the patient workflow \\ (plus number of department-specific workflows)}

Behavioral medicine services

Cardiac vascular services Critical care services

\section{2}

32 (with 8 unique workflows for labs; 2 for pharmacy; and 3 for physician documentation) 2 (with 5 unique workflows for scheduling)
10

3 5

2
Other applications and patient care technologies integrated with the EHR

\begin{abstract}
Applications used to manage frequent documentation of patient changes and to download data from hemodynamic monitoring, IV therapy and ventilator care devices. Application used to manage and track completion of diagnostic testing, therapy and care events.
\end{abstract}

Application used to manage health maintenance and preventive services reminders during a series of visits.

Table 2 (Continued) 
Table 2. Service lines with patient centered workflows and department-specific needs for workflow. (Continued)

\begin{tabular}{|c|c|c|}
\hline $\begin{array}{l}\text { Patient-centered workflows } \\
\text { with integrated processes }\end{array}$ & $\begin{array}{l}\text { Number of workflows used } \\
\text { with the patient workflow } \\
\text { (plus number of } \\
\text { department-specific } \\
\text { workflows) }\end{array}$ & $\begin{array}{l}\text { Other applications and } \\
\text { patient care technologies } \\
\text { integrated with the EHR }\end{array}$ \\
\hline Maternal-child services & 10 & \\
\hline $\begin{array}{l}\text { Oncology diagnostics } \\
\text { which includes research } \\
\text { and non research } \\
\text { chemotherapy and } \\
\text { radiation therapy }\end{array}$ & 2 & \\
\hline $\begin{array}{l}\text { Outpatient diagnostics } \\
\text { which includes radiology } \\
\text { and blood transfusions } \\
\text { Pediatrics services }\end{array}$ & $\begin{array}{l}6 \text { (with } 5 \text { unique } \\
\text { workflows for scheduling) }\end{array}$ & \\
\hline Pulmonary service & 1 & \\
\hline $\begin{array}{l}\text { Skilled nursing and acute } \\
\text { rehab services }\end{array}$ & 3 & \\
\hline $\begin{array}{l}\text { Surgical services which } \\
\text { include anesthesia }\end{array}$ & $\begin{array}{l}19 \text { (with } 5 \text { unique } \\
\text { workflows for scheduling) }\end{array}$ & $\begin{array}{l}\text { Application used to } \\
\text { manage the plan of care, } \\
\text { devices, supplies and track } \\
\text { specimens and results } \\
\text { during procedure. }\end{array}$ \\
\hline
\end{tabular}

Table 3. Surgical services patient-centered workflows

\begin{tabular}{ll}
\hline Workflow & Description of the workflow \\
\hline $\begin{array}{l}\text { Pre admission screening } \\
\text { phone all }\end{array}$ & $\begin{array}{l}\text { Describes the steps to document pre-procedure information } \\
\text { from testing, screening phone call and reviewing results. } \\
\text { Pre-anesthesia testing }\end{array}$ \\
$\begin{array}{l}\text { Describes the patient steps before surgery to participate in lab } \\
\text { testing, cardiac and/or pulmonary function tests as appropriate; } \\
\text { patient and family education; test result follow-up as }\end{array}$ \\
$\begin{array}{l}\text { appropriate. } \\
\text { Describes the patient and clinician steps in the preoperative } \\
\text { day surgery and inpatient }\end{array}$ \\
Day of surgery & $\begin{array}{l}\text { Describes the steps for patient arrival for surgery, recovery and } \\
\text { discharge from the surgical services. }\end{array}$ \\
Inpatient pre-admission & Describes the steps for a patient to be admitted after a surgical \\
& procedure when intended and unintended.
\end{tabular}


Table 3. Surgical services patient-centered workflows (Continued)

\begin{tabular}{ll}
\hline Workflow & Description of the workflow \\
\hline Pre-operative holding & $\begin{array}{l}\text { Describes the steps for preparing the patient for surgery and } \\
\text { anesthesia. } \\
\text { Describes the steps anesthesia providers would follow to care } \\
\text { for patients prior to, during and post anesthesia. } \\
\text { Anesthesia }\end{array}$ \\
Intra-operative & $\begin{array}{l}\text { nurse, technician) to follow during the intra-operative state of } \\
\text { a surgical case. } \\
\text { Describes the steps with patients recovering from anesthesia } \\
\text { preparing for ambulatory discharge and inpatient transport. }\end{array}$ \\
Inpatient care & $\begin{array}{l}\text { Describes the steps with patients in the hospitals through } \\
\text { discharge. } \\
\text { Describes the steps before, during and after a scope procedure } \\
\text { Endoscopy }\end{array}$ \\
& and steps for recovery and discharge. \\
\hline
\end{tabular}

workflows follow the patient, anesthesia provider, surgeon, post-anesthesia care unit nurse. The anesthesia provider and surgeon enter orders while the nurse medicates and intervenes to comfort patients within the inpatient recovery unit. The workflows continue to guide patient education and discharge planning steps for the patient. Post-

\section{Table 4. Department-specific workflows}

\begin{tabular}{ll}
\hline Workflow & Description of the workflow \\
\hline Surgery scheduling & $\begin{array}{l}\text { Describes steps to distribute surgery schedules to department, } \\
\text { units and clinics which support patients, family and significant } \\
\text { others. }\end{array}$ \\
Pescribes the steps followed by clinical staff within the \\
maintenance & $\begin{array}{l}\text { surgical department. } \\
\text { Case cart assembly }\end{array}$ \\
Describes the steps to assemble items needed for the patient's \\
surgical procedure. \\
Describes the steps the surgery department completes to \\
monitor charge entry and charge reconciliation. \\
Endoscopy specimen & $\begin{array}{l}\text { Describes the steps to process the specimen within the } \\
\text { Endoscopy Suite. } \\
\text { Describes the steps to maintain and facilitate pick lists. }\end{array}$ \\
Generating pick lists & $\begin{array}{l}\text { Describes steps to complete documentation during the } \\
\text { procedure for managing specimen verbal order and collection. } \\
\text { Intra-operative }\end{array}$ \\
Describes steps to complete maintenance of supply items \\
descriptions and items files that include devices purchased and \\
unused items.
\end{tabular}


discharge processes include quality monitoring, follow-up phone calls and/or follow-up in ambulatory clinic. All workflows include steps with and without technology support.

For any sites using an emergency service's events tracking application in late 2004, the health system began to change all emergency room services from modular workflows to patient-centered workflows. We took advantage of this change to get feedback on both modular and patient-centered types of workflows. With the input from multi-disciplinary teams from four additional hospitals in three states, in 2005, patientcentered workflows were designed and introduced for all services. The benefits of using the patient-centered workflows were obvious in the 2006 implementations of the EHRs. Far fewer issues were reported during the initial two weeks of implementation.

In addition, the issues and resolutions for EHR changes were identified at the system level and the organization level for 5 diagnostic related groups (see Table 5) from 2003 to 2006. Electronic documentation forms, security settings, and order sentences were changed more often at the system-wide level to address gaps, whereas the order sets and pharmacy were changed at the organization level. The clinical decision support, emergency tracking and the radiology system EHR applications were not changed. Not surprisingly, these last three applications had a set standardization across settings. As the level of standardization increased, the number of issues declined.

After 2005, the medication process including reconciliation (a component of the patient-centered workflow) became standard for all patient services and all organizations. This workflow includes the patient, nurse, attending and ordering physician, pharmacist, pharmacy technician, and respiratory therapist. When the drug data were missing or unclear, the workflow necessitated obtaining data from other records, previous caregivers, and the patient's pharmacies. When the nurse collects the essential information (e.g., allergies, height, weight, last dose taken) early in the workflow, the physician can order safely thereafter. Ordering physicians are encouraged to use EHR order sentences and order sets to facilitate accurate and complete order details and steps to monitor therapeutic or adverse effects. When the steps are taken, the ordering physician may see less interruption from alert screens. Pharmacists can be

Table 5. Health system and organization changes in electronic system for 5 diagnostic groups before and after the $6^{\text {th }}$ EHR implementation.

\begin{tabular}{lccccc}
\hline \multirow{2}{*}{ Applications } & \multicolumn{2}{c}{ System changes to EHR } & & \multicolumn{2}{c}{ Organization changes to EHR } \\
\cline { 2 - 3 } \cline { 5 - 6 } & Before & After & & Before & After \\
\hline Electronic forms & 23 & 5 & & - & - \\
Security & 8 & 0 & & - & - \\
Orders & 1 & 10 & & 1 & 0 \\
Order set & 3 & - & & 32 & 46 \\
Pharmacy & 1 & - & & 3 & 13 \\
Radiology & - & - & & - & - \\
ER tracking & - & - & & - & - \\
\hline
\end{tabular}

- No changes identified to the technology design and content design. 
consulted to review the patient's medications for therapeutic substitutions, to verify new medications, and to document dosing options to support the ordering provider.

In the core measures, the 2005 implementation site continues to report levels exceeding national averages. For seven surgical indicators, the site performs at a level $6.8 \%$ higher than the national average, with $89 \%$ of the surgery patients having received treatment for deep venous thrombosis (DVT) prevention within 24 hours before and after selected surgeries in 2009. The percent of patients receiving preventive antibiotics one hour before surgery was $98 \%$. Likewise, $94 \%$ of patients had stopped preventive antibiotics 24 hours after surgery. Individually, all seven indicators were better than the national percentile. The six acute myocardial infarction indicators were $8.8 \%$ higher with one not applicable. The pneumonia and heart failure indicators were also above the national average. The patient-centered workflows provided an engineering tool and processes for finding similarities that might simplify the patient processes for surgery. The inpatient and ambulatory workflows required uniform patient information in standard formats.

\section{DISCUSSION}

The redesign and integration of countless modular workflows evolved, between 2003 and 2005, out of feedback from earlier implementations. The positive experiences of later implementations, in 2006 through 2010, demonstrated greater standardization with cross functional patient-centered workflows, and less application-specific workflows. The patient-centered workflows were scripted with the patient, plus each clinician type and support department, and placed into role-based swim lanes ${ }^{1}$, to display interaction with each other. The swim lane for the patient included steps for sharing information about complaints, preferences, participation in testing, interventions, education, and (when involved) decisions. When teams of clinicians were asked to review the patientcentered workflows, they could view steps through the EHR functions, discuss options in daily practice that would reduce variation, and identify possible steps in the process focused on patient experiences to eliminate variation.

A post-implementation study can follow two basic approaches [23], either reductionist (taking apart the EHR applications that comprise an EHR) or holistic (assessing how different components work in complex unity). In 2005 interviews, clinicians requested a more holistic workflow representation, to integrate the EHR system. The processes were redesigned to reflect the perspective of the patient receiving service; from this perspective, clinicians come into and out of the patient's care (workflow). This became the use case for patient-centered workflows in EHR systems which others would support $[18,21]$.

To implement and advance workflows within the 37 hospitals, today, the health system reuses its inventory of standard patient-centered workflows. The same could be done for ambulatory clinics. The workflows continue to be reviewed and updated, and gaps are identified during cycles of change such as upgrades or during system wide

\footnotetext{
${ }^{1}$ A swim lane is an element used in process flow diagrams to distinguish each of the different clinicians and/or ancillary and supporting departments involved with the patient during a patient care workflow.
} 
process improvement initiatives. Brender [23] encourages this kind of dynamic process to evaluate the cycle of changes with HIT. The patient-centered workflows provide a patient perspective. Cross-functional flowcharts describe step-by-step information exchange between clinicians and each type of patient [22]. This provides a conceptual framework for evaluating an evolving EHR that must maintain a focus on patient services [18, 21] and involve clinical leaders [24].

EHR systems and patient-care technologies must precipitate re-engineering the care processes to improve daily workflows. In doing this, one must take a systematic and collaborative approach (to prevent re-inventing the wheel). The wisdom of the system must foster decisions to adopt best practices [25], and the redesign of processes should leverage people, process, technology and culture. Using these principles, Baty et al. [25] reported improved outcomes for underserved populations with diabetes mellitus. Similarly, Piontek et al. [26] found reduced mortality and length of stay from less adverse drug events.

In our experience, clinicians requested starting review of redesigned workflows a year before implementation and requested that we involve 25 percent of staff as super users, who should be educated on the EHR technology and content before starting process redesign. Nurses, pharmacists, therapists, physicians, and other support staff from rural and urban settings review process workflows and contribute to developing workflow interactions with patients. When participating, the clinicians viewed both patientcentered workflow and technology applications simultaneously during day long sessions [22] to identify gaps. The clinicians and staff wanted to know where and what to document, when and how to order, and why. The integrated patient-centered workflows were constructed to answer and guide teams of clinicians through processes, displaying EHR use, and collaborating on standard ways to re-engineer care for patient populations. In 2006, the implementations of the patient-centered workflows using EHR reduced the issues of clinicians' use of the technology dramatically. Subsequent implementations used the standardized workflows and have advanced the designs with smooth transitions.

The workflows improved standardization and fostered the clinical acceptance of the EHR. The standardization not only facilitated care transformation, record keeping, and billing but also allowed knowledge to be transferred from site to site. Before 2005, the patient's role had been missing in modular workflows, and therefore, the patient's story, and their communications and interactions with clinicians were not adequately captured or displayed. By asking multi-disciplinary groups who should do what, when, where, and why, we can display the patient's care with longitudinal records.

The longitudinal nature of EHRs will affect individuals, families and communities, challenging all to rethink the patient's role in decisions. Others have defined patientcentered care as recognizing the mind, body and spiritual needs and preferences of patients-informing and involving patients in decision making and self-management interventions $[6,28]$. To improve our system, in the future, we aim to understand how patients perceive their involvement in information gathering and what level of granularity is necessary for workflows and the exchange of this information.

The patient-centered workflows can advance use of patient care technologies and EHR systems, but clinicians must re-analyze the standardized workflows as health 
sciences improve and make the necessary readjustments of where, when and how to use patient and population information. Determining whether clinicians use or underuse technology should reveal gaps in EHR systems [1]. Analyzing workflows can also help us predict what information clinicians and patients will need to collect, and what information they will need to have available for decision making and for health information exchange. Inattention to workflows, over the long term, could lead to a decline in use over time and the development of work-around steps. Creating this culture of workflow understanding will sustain organizations through the inevitable upgrades and new technology implementation.

Our interviews precipitated clinician feedback on current practice, which created the impetus to change. The answers we collected contained new ideas using evidence-based practices to improve patient quality and safety designs within the EHR systems [22]. The discussion of care processes often precipitated the need to display the usability of EHR systems during meetings. When the clinician teams review patient-centered workflows, they identify the gaps in content and functionality, which leads to changes at the setting or system (see Table 5). The workflow representations are good methods for re-examining and improving care processes when implementing upgrades with the EHR [21, 22].

Finally, we agree with Guptill et al. [19], who stressed the importance of a culture, which promotes a knowledge-driven system; we believe such a knowledge-driven culture will embrace improved standardization (see Figure 2). Our goals for staff were simple: ensure care was not missed, bills were processed, safety was not compromised, and the best evidence-based practice protocols and knowledge were available for the end-user. The tools (workflows) to facilitate communication should not be underestimated. If done well, the system (a set of interrelated healthcare units) should engage in joint problem solving [26] and focus on standardizing communication to create a new workflow that provides care from a longitudinal perspective rather than an episodic or modular perspective.

\subsection{Study Limitations}

In 2005, the technology did not adequately support the nursing or physician workflow for care coordination and documentation. Neither a nursing framework, such as Gordon's functional health patterns [20], nor the nursing practice taxonomy for assessment [27] was comprehensively used as an assessment framework for care planning within the electronic documentation infrastructure. Until 2008, nursing care was planned using some paper, an inefficient system for nurses who coordinated care using a mix of paper and electronic records. The new technical functions were designed to link processes for nursing assessments, diagnoses, outcomes and interventions to allow interdisciplinary plan of care within daily practice and eliminate paper records. Closer attention to meeting the needs of these nursing processes should improve opportunities to enhance safety and quality of patient-centered services.

The study focuses on one health system with multiple implementations, and maintaining the knowledge resource for the workflows. The technology upgrades altered functionality, so we were limited in our ability to control for these variables. Due to the 
upgrades between setting implementations, the health system was challenged in reaching a level of standardization with patient-centered workflows. The workflows were reused and the changes from setting to setting did result in a common workflow that was placed in the inventory of knowledge resources. The mere use of common workflows from setting to setting reduced the opportunity for work-arounds. The reuse of these workflows would be challenging for anyone wishing to focus solely on the technology because the clinicians' contributions resulted in workflows that were not dependent on just technology. The holistic patient perspective required full service workflows.

\section{CONCLUSION}

Adoption of patient-centered workflows has been increasing, and standardization in all types of settings would be beneficial for patients. As requested by clinicians, we designed integrated, cross-functional, patient-centered workflows that focus on patient care; these workflows phased out the multiple, isolated, application-specific, modular flowcharts. Patient-care experts helped incorporate ways to capture the participation of the patients and their families, so that nothing was left undiscovered in daily workflow. Without the patient/family information contributions to information within EHR systems, clinicians are unable to make full use of the longitudinal use of the health information technologies that can influence care decisions.

In addition to creating patient-centered, role-based workflows, we created new roles in the organization and a systematic method to introduce the new process flows. The roles of process liaison, service line champion, and super user are all essential in achieving a successful implementation. These roles are created early in the 14 month preparation process and are taught not just EHR functionality but the patient-centered process workflows. This culture of process understanding and design is then sustained throughout the implementation and into future hospital improvement efforts.

\section{ACKNOWLEDGEMENTS}

The authors acknowledge the support of Trinity Health - Novi, Michigan home office and all of the organizations involved with the evolution of patient-centered workflows and clinical workflows.

CONFLICT OF INTEREST: SO and JMK had a work-related financial interest in Trinity Health - Novi who funded this study of the clinicians' feedback on the review process to prepare for implementation and the knowledge resource to maintain the workflows. This interest did not affect the design, interpretation, or reporting of any of the study herein.

\section{REFERENCES}

[1] Andre B, Ringdal GI, Loge JH, Rannestad T, Kaasa S. The importance of key personnel and active management for successful implementation of computerized-based technology in palliative care. Computers, Informatics, Nursing, 2008, 26(4), 283-289.

[2] Ash JS, Berg M, Coiera E. Some unintended consequences of information technology in health care: the nature of patient care information system-related errors. J. Am Med Inform Assoc, 2004, 11, $104-112$. 
[3] Ash JS, Sittig DF, Poon EG, Guappone K, Campbell E, Dykstra RH. Categorizing the unintended sociotechnical consequences of computerized provider order entry. Int J Med Inform, 2007, 76, S21-S27.

[4] Campbell EM, Sittig DR, Ash JS, Guappone KP, Dykstra RH. Types of unintended consequences related to computerized provider order entry. J Am Med Inform Assoc, 2006, 13, S47-S56.

[5] Harrison MI, Koppel R, Bar-Lev S. Unintended consequences of information technologies in health care - an interactive sociotechnical analysis. J Am Med Inform Assoc, 2007, 14(5), 542-549.

[6] Maizes V, Rakel D, Niemiec C. Integrative medicine and patient-centered care. Explore, 2009, 5(5), 277-289.

[7] Kohn LT, Corrigan J, Donaldson MS. To Err Is Human: Building a Safer Health System, National Academic Press, Washington, D.C., 2000.

[8] Santell JP, Kowiatek JG, Weber RJ, Hicks RW, Sirio CA. Medication errors resulting from computer entry by nonprescribers. American Journal of Health-System Pharmacy, 2009, 66 (9), 843-853. PMID: 19386948

[9] Lohr S. Government wants to bring health records into computer age. New York Times, 2004, July 21, 2004: Business day.

[10] Gerstle RS. Electronic prescribing systems in pediatrics: the rationale and functionality requirements. American Academy of Pediatrics, Council on Clinical Information Technology; Pediatrics, 2007, 119 (6), 1229-1231.

[11] Stewart BA, Fernandes S, Rodriguez-Huertas E, Landzberg M. A preliminary look at duplicate testing associated with lack of electronic health record interoperability for transferred patients. J Am Med Inform Assoc, 2010, 17 (3), 341-344.

[12] Kazemi A, Fors UG, Tofighi S, Tessma M, Ellenius J. Physician order entry or nurse order entry? Comparison of two implementation strategies for a computerized order entry system aimed at reducing dosing medication errors. Journal of Medical Internet Research, 2010, 12 (1), e5 doi: 10.2196/jmir.1284, URL: http://www.jmir.org/2010/1/e5/.

[13] Szekendi MK, Sullivan C, Bobb A, Feinglass J, Rooney D, Barnard C, Noskin GA. Active surveillance using electronic triggers to detect adverse events in hospitalized patients. Quality \& Safety in Health Care, 2006, 15 (3), 184-190.

[14] Thompson D, Johnston P, Spurr C. The impact of electronic medical records on nursing efficiency. Journal of Nursing Administration, 2009, 39 (10), 444-451.

[15] Malhotra AK, Martin N, Jacoby M, Tarrant J, Guilford K, Wolfe LG, Aboutanos MB, Duane TM, Ivatury RR. What are we missing: results of a 13-month active follow-up program at a level I trauma center. Journal of Trauma, 2009, 66 (6), 1696-1703.

[16] Deckelbaum DL, Feinstein AJ, Schulman CI, Augenstein JS, Murtha MF, Livingstone AS, McKenney MG. Electronic medical records and mortality in trauma patients. Journal of Trauma, 2009, 67 (3), 634-636.

[17] Leadership . Transforming Care Delivery. A Publication of HFMA Learning Solutions, Inc. A Subsidiary of the Healthcare Financial Management Association, 2009, Retrieved on June 1, 2010 at www.trinity-health.org/contentportal/groups/public/.../cportal_005712.pdf

[18] Boaden R, Joyce P. Developing the electronic health record: what about patient safety? Health Services Management Research, 2006, 19(2), 94-104.

[19] Guptill J. Knowledge management in health care. J Health Care Finance, 2005, 31(3), 10-14.

[20] Gordon M. Manual of nursing diagnoses, 12th ed., Mosby, St. Louis, 2010.

[21] Unertl KM, Weinger MB, Johnson KB, Lorenzi NM. Describing and modeling workflow and information flow in chronic disease care. Journal of the American Medical Informatics Association. 2009, published August 28 as dol:10.1197/jamia.M3000.

[22] Brokel JM, Harrison MI. Redesigning care processes using an electronic health record: A system's experience. The Joint Commission on Quality and Patient Safety, 2009, 35(2), 82-92. 
[23] Brender J. Handbook of Evaluation for Method for Health Informatics., Academic Press, New York, 2006, 37-73.

[24] Geibert RC. Using diffusion of innovation concepts to enhance implementation of an electronic health record to support evidence-based practice. Nurs Adm Q, 2006, 30(3), 203-210.

[25] Baty PJ, Viviano SK. Schiller MR, Wendling AL. A systematic approach to diabetes mellitus care in underserved populations: improving care of minority and homeless persons. Family Medicine, 2010, 42(9), 623-627.

[26] Piontek F, Kohli R, Conlon P, Ellis JJ, Jablonski J, Kini N. Effects of an adverse-drug-event alert system on cost and quality outcomes in community hospitals. Am J Health Syst Pharm, 2010, 67(8), 613-620.

[27] Von Krogh G, Dale C, Nåden D. A framework for integrating NANDA, NIC, and NOC terminology in electronic patient records. Journal of Nursing Scholarship, 2005, 37(3), 275-281.

[28] Agency for Healthcare Research and Quality. Expanding patient-centered care to empower patient and assist providers, 2009, Retrieved from AHRQ website on August, 30, 2009 at http://www.ahrq. gov/qual/ptcareria.htm 


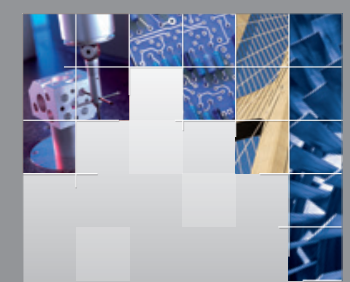

\section{Enfincering}
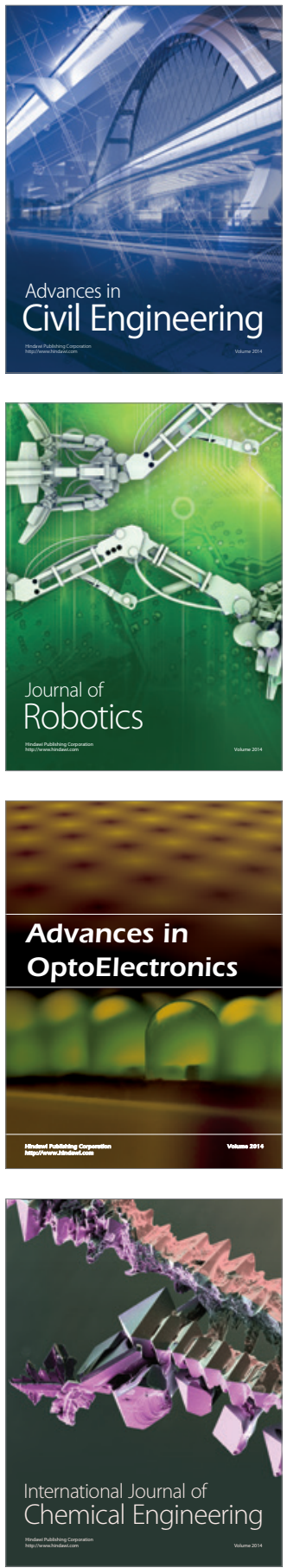

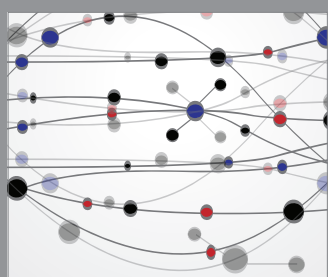

The Scientific World Journal

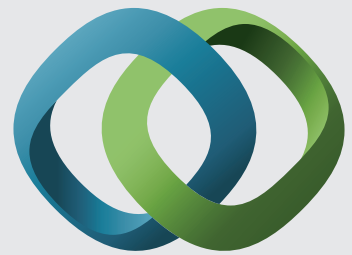

\section{Hindawi}

Submit your manuscripts at

http://www.hindawi.com
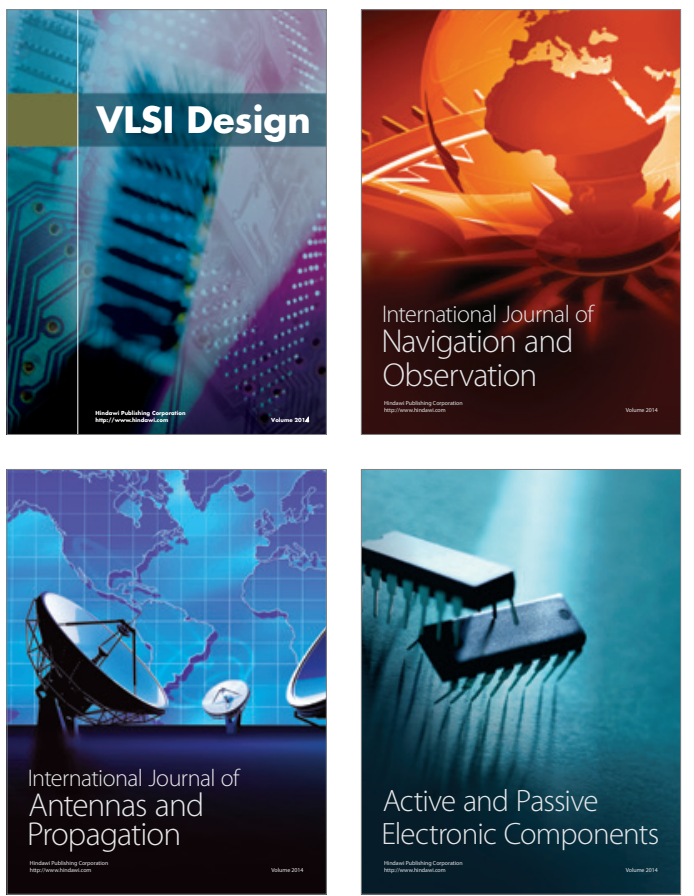
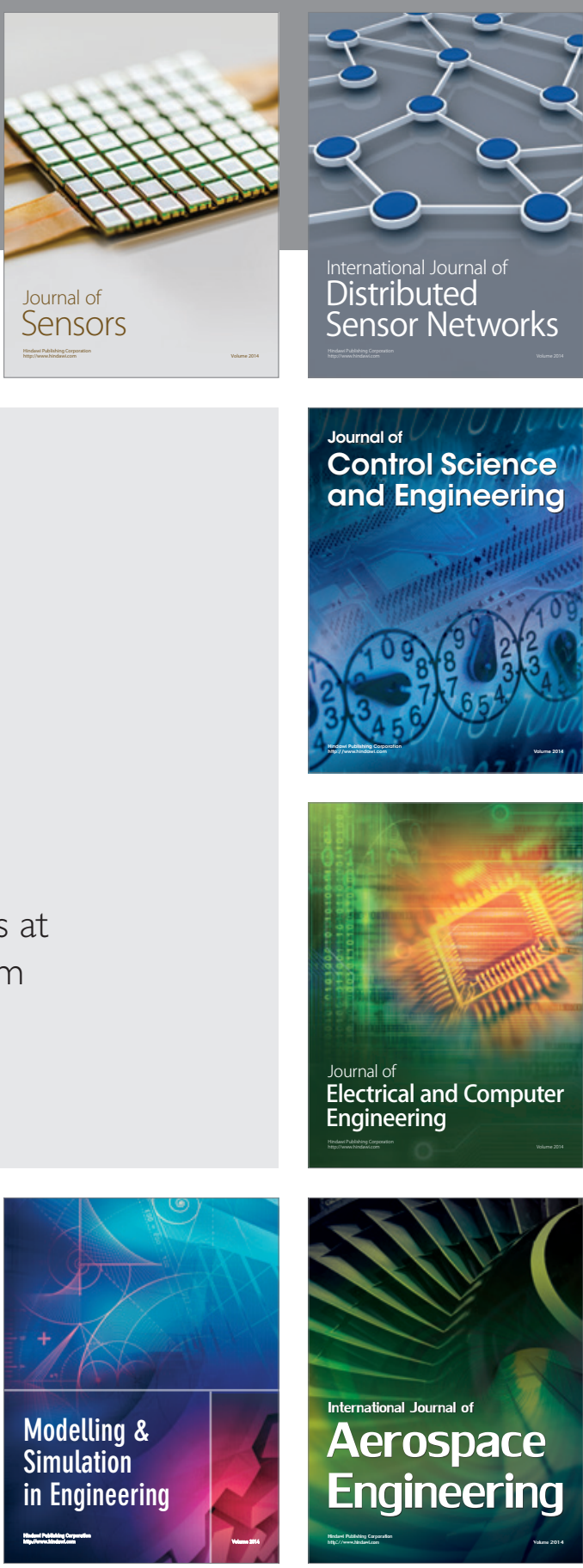

International Journal of

Distributed

Sensor Networks

Journal of

Control Science

and Engineering
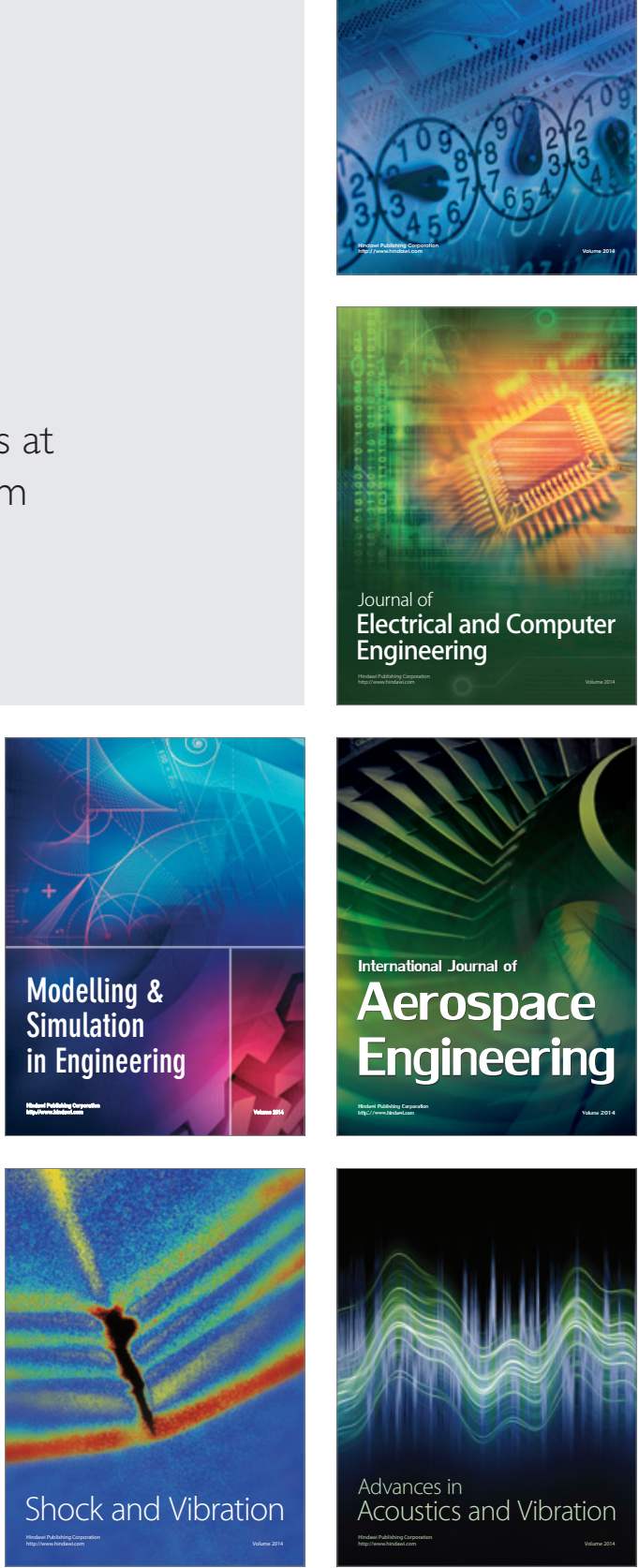